\title{
Multiscale Modeling of Vibration Damping Response of Shape Memory Polymer Fibers
}

\author{
Soodabeh Sharafi ${ }^{\mathrm{a}}$, Guoqiang $\mathrm{Li}^{\mathrm{a}}$,b,* \\ a Department of Mechanical \& Industrial Engineering, Louisiana State University, Baton Rouge, LA 70803, \\ USA \\ ${ }^{\mathrm{b}}$ Department of Mechanical Engineering, Southern University, Baton Rouge, LA 70813, USA
}

"Correspondence author: Tel.: 001-225-578-5302; Fax: 001-225-578-5924; E-mail: lguoqi1@1su.edu

\begin{abstract}
Health of a structure under vibration loads is highly related to the damping characteristics of the system. This work explores an engineered smart Shape Memory Polymer Fiber (SMPF) system that is capable of adjusting its damping capabilities based on applied load frequency and temperatures. A SMPF based structure with smart vibration/damping capability is of interest to many industries including aerospace, automotive and biomedical sectors. SMPFs enable structure engineers to incorporate smartness functionality into their design through programming or training of SMPFs. While SMPF structural applications in the case of static loadings have been studied, the application of SMPFs in mitigating vibration responses of a structure has not been fully addressed in the research arena. The vibration damping response of a SMPF material system is studied with a goal to design damping response of smart structures that can mitigate severe vibrations. In this work vibration damping response of a SMPF bundle is experimentally studied through Dynamic Mechanical Analyzer (DMA) machine, and a numerical model is developed to correlate the loss/storage moduli to the damping/stiffness characteristics of the SMPF system. The model is then applied to study forced vibration responses of SMPFs. DMA data are utilized to verify the performance of the proposed model. The presented experimental data and the numerical model provide insight into vibration damping application of SMPFs in smart structures.
\end{abstract}

Keywords: A: Polymer Fibers; A: Smart Materials; B: Vibration; C: Analytical Modeling.

\section{Introduction}

Shape memory polymers are classified as smart materials that are able to respond to the external stimuli sources such as heat, sound, light, etc. [1-7]. Most recently, Li and 
co-workers have developed several Shape Memory Polymer Fiber (SMPF) reinforced polymer matrix composite (PMC) systems with capabilities for crack self-healing [8-14]. Most of the up to date studies concern quasi-static response of shape memory polymers including shape memory polymer fibers and artificial muscles [15-37]. However, there is currently a lack of modeling technique to addresses vibration analysis of SMPFs.

Vibration analysis enables designers to study stability of the structure when oscillation occurs. Unwanted noises or dissipative vibration mechanisms, resulted from lack of meticulous design of components, may lead to premature failure of structural components. Prognosis techniques and damping of severe oscillating loads become a cardinal point in the structural design. For the past decades, the inception of new technologies such as space exploration programs have provided an increasing need for lightweight materials with high damping capabilities and tolerance to the harsh environments.

The nature of damping lies in the microscopic response of a material when vibration is initiated. Damping property of polymers is strongly temperature dependent, especially around the glass transition temperature where polymers hit the highest level of damping [38]. Elevated damping capability is related to the excessive viscoelastic motion of polymer chains in this temperature. In fact, these giant molecules transform from low temperature solid-like state to high temperature liquid-like state. During this reversible transition, the long range molecular reordering results in dissipation of the energy of vibration [39]. Crandall discussed the nature of some important damping mechanisms to clarify their dependency on the amplitude and frequency of a cyclic motion [40].

As compared to conventional non-shape memory polymers, SMPs experience about two-orders of change in stiffness in the glass transition region, signifying exceptional molecular viscoelastic motion, and thus potentially superior damping properties. It is expected that if the SMP fibers are used in fiber reinforced polymer composites, the damping properties of the composites can also be enhanced. The damping properties of fiber reinforced polymer composites have been a topic of intensive research in the recent years [41-48].However, before the wide application of SMP fibers in polymer composite for vibration damping, understanding of the damping behavior and effect of materials parameters on the damping performance of SMP fibers, particularly 
through mathematical modeling, is highly desired.

The modeling techniques are an essential part of the vibration analysis in order to minimize the experimental trial-and-error efforts for optimizing damping capability of a structure. Four distinct categories of modeling techniques are introduced by Treviso et al., including linear viscoelastic, complex modulus, strain energy methods and dissipative coordinate methods [41]. In linear viscoelastic models, material is modeled through application of spring elements, the representation of elastic segment, and the dashpot elements, the representation of inelastic or dissipative part. Nowick et al. have elaborated the application of this methodology to predict dynamic response of inelastic solids; however, the nature of energy dissipation in this type of modeling remains unanswered [49]. Complex modulus methodologies are the second category of these modeling techniques. However, their cumbersome computations hinder their applications. Crandall has elaborated some examples to illustrate application of the proposed mathematical formulations [40]. In another study, Bagley and Torvik have suggested that damping mechanism results from a frequency dependent modulus, which is a fractional power of frequency. Also, Caughey et al. applied Raid's non-linear model to describe hysteric damping mechanism. The proposed model suggests a mathematical procedure to include nonlinearity of dynamic response. However, discrepancy between the proposed model and the exact solution below the resonance frequency is merely negligible [50]. The biggest drawback, for these types of modeling as mentioned above, is the complexity associated with the definition of viscoelastic behavior [51].

The third category of modeling is based on an exchange of strain energy for vibration analysis. Johnson et al. have used the notation of change in the strain energy density to measure the existence of deformation mechanism related to loading and unloading condition. The strain energy methods suffers from lack of direct methodologies to relate material parameters to the experimental results; this paucity has overshadowed their applications [52].

The fourth and last category encompasses modeling techniques, which are based on the evolution of internal variables; these methodologies are not easily supported by the experimental results. In this category, the notable Golla-Huges-McTavish (GHM) method is formulated through application of the second-order matrix to solve the equation of 
motion. The material properties are substituted to mass, stiffness and damping matrices. The so-called "auxiliary coordinate" has been introduced to incorporate the internal dissipation mechanism $[40,53]$. Lack of accuracy in the introduced curve fitting parameters obfuscates their unlimited applications. However, to compensate for the limited applications of the proposed model, McTavish et al. have suggested a new approach to modify these curve fitting parameters [54]. There are few other methodologies that are in close relation to the GHM method such as augmenting thermodynamic fields (ATF) and anelastic displacement field (ADF) $[55,56]$. ATF is a coupled equation of motion demanding the adaptability of the thermodynamic field at each element. ADF is proposed to simplify the ATF methodology by considering the evolution of "displacement field" introduced to solve the higher order equation of motion. Despite their sophisticated methodology, correlation to the experimental results is hardly reachable.

As a new member of the smart materials family, there are few studies concerning the application of shape memory polymer fibers, SMPFs, either independently or as a reinforcing element to augment dynamic material property of a composite system. Therefore, in this work we aim at exploring the possibility of design and development of smart SMPF systems to control the energy dissipation and suggest the optimum range of damping properties, which can be programmed at different temperatures. Also we propose a novel numerical methodology to correlate dynamic mechanical analysis (DMA) results to the stiffness and damping coefficient of the Single Degree of Freedom (SDOF) equation of motion. Both experimental tests and numerical modeling analysis are incorporated to fully understand the vibration damping response of SMPFs. DMA test is utilized to study the complex viscoelastic behavior of SMPFs; frequency sweep tests are carried out to obtain the storage and loss moduli of SMPF bundles at various temperatures. A phenomenological SDOF model is then developed based on the DMA test results to calibrate the two proposed relations. It is shown that the proposed modeling procedure can favorably capture the forced vibration response of the SMPF system at different temperatures. These proposed relations are meant for relating the micro structural properties of polymer chains to the macroscopic vibration/damping response.

This work is organized as follows, first the theory behind the forced vibration 
analysis is briefly discussed and then the SDOF model is described. After that, the experimental setup and test results are presented; furthermore, multiscale model and its numerical implementation procedure are elaborated in two sections. Finally, the results and discussions are presented.

\section{Single Degree of Freedom (SDOF) Model for SMPF System}

The proposed SDOF model for SMPF system is discussed in this section and the performance of the developed model is examined in Section 6 where the reported experimental data, which concern with forced vibration of a typical SMPF sample under sinusoidal forces, together with the model performance are shown. It is assumed that the vibration response of the SMPF sample is described through a SDOF model. The steady state SDOF equation of motion reads:

$m \ddot{x}+c \dot{x}+k x=F_{0} e^{i \omega t}$

where $m$ is the mass of a vibrating fiber, $c$ is the coefficient of damping, $k$ is the stiffness of a typical SMPF sample, and $F_{0}$ is the amplitude of the applied force.

Steady state response of the linear system to the harmonic force could be expressed through definition of a transfer function $\mathrm{H}(\mathrm{t})$ and a corresponding phase angle which is well-established in the literature and is given by [57]:

$x(t)=\frac{F_{o}}{k} H(\omega) e^{i(\omega t-\phi)}$

where $\omega$ is the frequency, $H(\omega)$ is called transfer function or magnification factor, and $\phi$ is the phase lag.

$\phi=\tan ^{-1}\left(\frac{2 \zeta r}{1-r^{2}}\right)$
$H(\omega)=\frac{1}{\sqrt{\left(\left(1-r^{2}\right)^{2}+(2 \zeta r)^{2}\right.}}$

in which $\zeta=\left(\frac{c}{c_{c}}\right)$ is loss factor, $c_{c}=2 m \omega_{n}$ is the critical damping, and $r=\frac{\omega}{\omega_{n}}$ is the ratio of the frequency over natural frequency.

For studying the vibration damping of SMPF sample, it is assumed that the mass of the system is constant. In order to address the temperature and frequency dependence, the coefficient of damping $c$ and stiffness $k$ are formulated systematically in the following. It is trivial to relate the stiffness $k$ of the SMPF system to the storage modulus $E^{\prime}$ and the damping coefficient $c$ to the fraction of the loss modulus $E^{\prime \prime}$ through 
experiments. Based on our simulation results, we have found that the classical stiffnesstensile modulus correlation provides a good relationship, if the dynamic storage modulus is used instead of Young's modulus:

$k=\frac{E^{\prime} A}{L}$

where $A$ and $L$ are area and length of the typical SMPF sample.

In the case of the underdamped systems with $\xi<1$, the relationship between the damping coefficient $c$ and loss modulus $E^{\prime \prime}$ reads:

$c=c_{c} \times \frac{E^{\prime}}{E^{\prime \prime}}$

where $c_{c}=2 m \omega_{n}$ is the critical damping which is a function of the natural frequency of a system, and the natural frequency $\omega_{n}=\sqrt{\frac{k}{m}}$ depends on stiffness and mass of an oscillatory system.

The performance of Eqs. (5) and (6) are examined in Section 6, where these relations are utilized to study the forced vibration responses of the SMPF system. The next step is to formulate the temperature and frequency dependent $k$ and $c$. In order to fulfil this task the temperature and frequency dependent $E^{\prime}$ and $E^{\prime \prime}$ functions are formulated and Eqs. (5) and (6) are utilized to find the temperature and frequency dependence of $k$ and $c$. With this strategy the frequency and temperature DMA sweep tests can be incorporated to calibrate the temperature and frequency effects.

One may note that $E^{\prime}$ and $E^{\prime \prime}$ are strong functions of temperature and they change drastically near glass transition temperature $T_{g}$. To include the temperature effect in the model the following linear relations are utilized:

$E_{T}^{\prime}= \begin{cases}E_{E G T}^{\prime}-m_{1}\left(T-\left(T_{g}+\sigma_{g}\right)\right) & T<\left(T_{g}+\sigma_{g}\right) \\ E_{E G T}^{\prime}-m_{2}\left(T-T_{h}\right) & T>\left(T_{g}+\sigma_{g}\right)\end{cases}$

$m_{1}=\frac{\left(E_{0 T}^{\prime}-E_{1 T}^{\prime}\right)}{\sigma_{g}}, m_{2}=\frac{\left(E_{E G T}^{\prime}-E_{h T}^{\prime}\right)}{\left(T_{h}-T_{g}\right)}$

$E_{T}^{\prime \prime}=\left\{\begin{array}{lc}E_{E G T}^{\prime \prime}-n_{1}\left(T-\left(T_{g}+\sigma_{g}\right)\right) & T<\left(T_{g}+\sigma_{g}\right) \\ E_{h T}^{\prime \prime}-n_{2}\left(T-T_{h}\right) & T>\left(T_{g}+\sigma_{g}\right)\end{array}\right.$

$n_{1}=\frac{\left(E_{0 T}^{\prime \prime}-E_{E G T}^{\prime \prime}\right)}{\sigma_{g}}$, and $n_{2}=\frac{\left(E_{E G T}^{\prime \prime}-E_{h T}^{\prime \prime}\right)}{\left(T_{h}-T_{g}\right)}$

where $E_{0 T}^{\prime}$, and $E_{0 T}^{\prime \prime}$, are respectively storage modulus and loss modulus at room temperature (below $\mathrm{T}_{\mathrm{g}}$ region), $E_{E G T}^{\prime}$, and $E_{E G T}^{\prime \prime}$ are respectively storage modulus and 
loss modulus at the end of transition event and $E_{h T}^{\prime}, E_{h T}^{\prime \prime}$ are storage modulus and loss modulus at high temperature (above $\mathrm{T}_{\mathrm{g}}$ region), respectively, and $T_{g}$ and $\sigma_{g}$ are respectively glass transition temperature and half of the bandwidth of the transition event. Coefficients $m_{1}, m_{2}, n_{1}$, and $n_{2}$, which are all obtained directly from DMA test data, correspond to the load carrying and damping capabilities of the SMPF sample.

To include the frequency effects on the loss and storage moduli, the two following relations are developed to capture frequency dependent response:

$E_{f}^{\prime}=E_{1 f}^{\prime} f^{\beta}$

$E_{f}^{\prime \prime}=E_{0 f}^{\prime \prime}\left(f-f_{E}\right)^{\gamma}+E_{0 f}^{\prime \prime}(f)^{\gamma}-E_{0 f}^{\prime \prime}$

where $E_{0 f}^{\prime \prime}$ is the loss modulus at low frequencies, $f_{E}$ is the upper limit of the frequency response and $\gamma$ corresponds to the loss factor, viz. $\tan \delta$, at the highest frequency available in a set of experiment; $E_{1 f}^{\prime}$ is the storage modulus at median frequency in a set of experiment, and $\beta$ is the corresponding loss factor at frequency, $f$.

It is worth noting that all parameters in Eqs. (7) - (10) are directly obtained from the DMA test results. Eventually, the temperature and frequency dependent storage and loss moduli of the SMPF system can be expressed as

$E^{\prime}=E_{f}^{\prime} \times E_{T}^{\prime}$

$E^{\prime \prime}=E_{f}^{\prime \prime} \times E_{T}^{\prime \prime}$

\section{Experimental Setup and DMA Test Procedures}

The specimens were in the form of bundles of SMPF, and each bundle contained one hundred filaments and was fixed together by the fiber geometry clamp in tension mode, which is a fixture of the testing instrument. Microstructure of these fibers consists of two phases that called "soft and hard domains". The chemical composition of the SMPF soft domain is polybutylene adipate (PBA), and hard domain is 4'4diphenylmethane diisocyanate (MDI) and 1, 4-butanediol (BDO). The length of the typical SMPFs specimens ranged from $10 \mathrm{~mm}$ to $11 \mathrm{~mm}$. The cross-section area of a typical sample is $2.25 \times 10^{-4} \mathrm{~cm}^{2}$. The length and diameter of the samples were measured separately.

The isothermal strain controlled frequency sweep tests were conducted, using TA Instruments RSA-G2, for two typical SMPF bundle samples. Dynamic mechanical testing 
was used to characterize the dynamic result of the typical SMPFs at controlled frequencies $(0.01 \mathrm{~Hz}-100 \mathrm{~Hz})$ at two temperatures $\left(25\right.$ and $\left.50^{\circ} \mathrm{C}\right)$. Storage modulus, loss modulus, $\tan \delta$ and displacement were measured at each frequency.

DMA test is one of the most famous sinusoidal oscillatory mechanical tests; it is used to identify the dynamic mechanical property of shape memory polymer fiber at different temperatures. At each frequency, the stress input along with the corresponding strain output is recorded. ASTM D4092 standard is utilized to determine the testing procedure for the shape memory polymer fiber. The typical responses offered by DMA test instruments are storage modulus, E', and loss modulus, E". The storage modulus is correlated to the elastic response of the material and the loss modulus represents the capacity to dissipate energy of vibration observed during loading. The ratio of loss modulus over storage modulus is called loss tangent $(\tan \delta)$."

Step time is the time during a particular step at which the measurement was completely calculated. After passing this time, the macromolecule reach to a state of dynamic equilibrium in which output data are recorded. The step time depends on temperature, composition and frequency of the applied loading. Oscillation force is an output parameter from DMA that indicates the maximum force due to the applied oscillation. A static force (preload) is applied at the beginning of the test to avoid fiber buckling during the tests.

\section{Vibration Damping of SMPFs in terms of Molecular Mechanisms}

Desired properties of SMPs could be tailored through engineering cross-links and microstructures in order to obtain superior properties such as high damping and stiffness. Two main molecular level mechanisms, which significantly contribute to the damping of polymers, are chain segmental motion and conformational rotation, which are augmented during the glass transition event. Below Tg, chain conformational rotations are frozen; therefore, polymer molecules show higher storage capabilities while the loss modulus is lower than that in the glass transition region. The applied dynamic energy during vibration perturbation produces dissipative heat. This generated heat results in two damping mechanisms 1) activating entropy driven forces when temperature is below $\mathrm{Tg}$ and 2) structural relaxation mechanism when temperature is higher than $\mathrm{Tg}$. These mechanisms are elaborated in the following paragraphs. In the case of high frequency or 
high temperature conditions, where polymer chains are highly mobile, the increased mobility of chains may lead to the fatigue initiation as reported by Dao [58].

- SMPF microstructural damping

The polyurethane SMPF in the microscopic scale consists of soft and hard domains [59]. The soft and hard domains respond differently when mechanical stress is applied. The soft domains are made of a number of randomly distributed chains while the hard domains consist of highly oriented polymer chains which connects chains of soft domain and strengthens the SMPF. Therefore, the soft domains are more flexible when undergo mechanical loading; chains inside the soft domains tend to rearrange into a more ordered structure at temperatures below the glass transition temperature. The glass transition of the soft domain is around $-55^{\circ} \mathrm{C}$ and the hard domain is observed around $110^{\circ} \mathrm{C}$. However, the glass transition temperature of the mixture of the soft and hard domains is $25^{\circ} \mathrm{C}$, on which our analysis is based, and half of the bandwidth of the glass transition event, $\sigma_{g}$, is also $25^{\circ} \mathrm{C}$ which is imported from experiment.

- Temperature effect on SMPF damping

Vibration analysis of a typical SMPF sample shows that chains are completely capable of restoring their original shape below the glass transition of the soft domains, $T_{g_{s}}$. Upon further heating, the increased mobility of the soft chains would activate the structural relaxation mechanism that is saturated at glass transition of hard segment, $T_{g h}$. On the other hand, the compliance of the hard segments starts to increase from temperature near $T_{g h}$ and hits the apex beyond $T_{g h}$. Consequently a typical SMPF sample shows higher damping capabilities beyond $T_{g h}$ due to the increased mobility. Figure 1 shows a schematics of damping property changes with the temperature and microstructural $\mathrm{T}_{\mathrm{g}}$. Based on volume fractions of hard and soft domains, the damping of SMPF system can be varied between two extreme cases of hard and soft cases. 


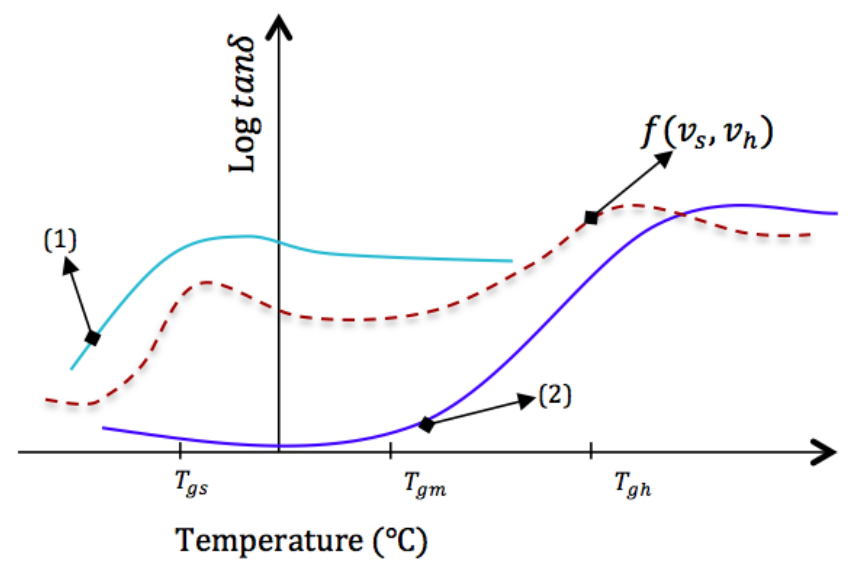

Figure 1 schematic representation of effect of microstructure on the damping property of SMPF changing with temperature. (1) The damping capability of Soft domains which hit the apex in the $T_{g s}$,(2) The damping capability of hard domains and dashed line shows damping capability of mixture of two domains which depends on the volume fraction of each domain.

- Frequency effect on SMPF damping

At the glassy region when the SMPF is disturbed at low frequencies, the internal friction among amorphous chains (originate from bond motions) activates the energy absorption mechanisms. The bonds liberation and side chains motion will be augmented after the soft domain absorbs the energy of vibration. Further accumulation of these microscopic motions would later trigger the slip condition in which the relative motion of domains would be initiated. The overall damping is still estimated negligible because slippage and other motions are still frozen in the glassy region.

At higher frequencies the frictional forces at the soft and domain boundaries produce damping effects. The physics behind these frictional forces relies in the disparity between the viscose response of soft and hard domains. As a result of these incompatible viscose motions between hard and soft domains, a sliding event that is a dissipative mechanism occurs at the boundary layer between the hard and soft domains. At more advanced frequencies, due to the alleviated dissipative mechanisms the temperature of SMPF may pass $T_{g h}$. That leads to higher chains mobility and higher damping capability. On the other hand, fatigue in the polymer chains may hinder to achieve higher damping levels [58]. Figure 2 represents a schematic of frequency effect on the damping properties in which lower damping at high frequencies is associated with the fatigue effects. 


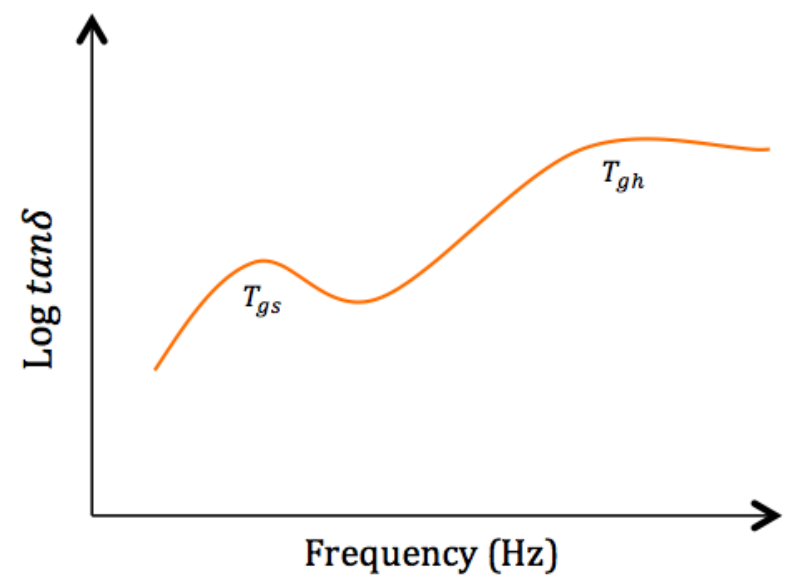

Figure 2 Schematic representation of the effect of microstructure on the damping property of SMPF changing with frequency.

The damping capability changes with temperature and frequency. Higher frequencies activate more segments of polymer chains and increase the restoring force viz. entropic force; this force supports chains to obtain their random coil configuration. The higher entropic force results in higher storage modulus. And therefore, highest energy of vibration would be damped.

\section{Numerical Approach}

To solve the equation of motion the Runge-Kutta approach is adopted in this work. Runge-Kutta methodology provides higher degree of precision than typical Euler approach in solving second order differential equations. The fourth order Runge-Kutta is applied to obtain the time domain representation of the equation of motion. Typical algorithm for finding the solution for first order ODEs contains four auxiliary quantities which should be calculated at each step and then the new value for the function should be updated based on these quantities [60]. The numerical procedure to obtain the stiffness and damping and to calculate the resulted displacement is given as follows:

1- Input $F, \omega$

2- Calculate $E^{\prime}$ and $E^{\prime \prime}$ from Eqs. (7) and (8)

3- Calculate stifness and damping from Eqs. (5) and (6)

4- Replace k and c in the Eq. (1)

5- Compute $x(t)$ from Eqs. (2), (3) and (4)

6- Compute error, e, of $x(t)$ (modeling compared to experiment) 
7- Modify $\mathrm{k}$ by incorporating the error $k_{i+1}=k_{i} \times(1+e), k_{i}$ is the latest damping coefficient

8- Go to the steps 4,5 , and then 6

9- If the error e $<5 \%$ then Go to Step 12

10 - If the error e $>5 \%$ GO to Step 11

11- Replace $\mathrm{c}$ by $c_{i+1}=c_{i} \times(1+e)$ where $c_{i}$ is the latest damping coefficient

12-Compute $x(t)$ and compare with experimental results

13- Display $\mathrm{c}$ and $\mathrm{k}$

The optimization process which is part of the numerical steps is crucial especially for frequencies over $60 \mathrm{~Hz}$. The importance of this step is to reduce the error associated with modeling the experimental results related to the storage moduluss and loss modulus in Fig. 3 and Fig. 4. Once the viscous damping coefficient, c, and stifness, k, are calculated from experiments and pluged into the equation of motion, the resulting displacement is obtained and is shown in Fig. 8 as square symbols; the results before optimization process provides an avarage error about $15 \%$ where the majority of this discrepancy is related to the fact that the model's stifness and damping coefficient carry errors at higher frequencies. However, through the optimization process, step 7, the stiffness is modified by incorporating error of calculations. The result of this modification is shown in Fig. 6 with circles. The coresponding result elucidates that at higher frequencies, SMPF is in rubbery elasticity, i.e. in softenning event; however, the model indicates continuous leathery response, i.e. stiffer structure at higher frequencies, which produces errors in calculations. As a result of error mitigation process, the average error among actual results and simulation outcome is reduced to $8 \%$. The accpetable error margine in the majority of typical engineering designs is $5 \%$. The reduced error is related to the effect of damping coefficeint, where in reality the capability of the specimen drops more drastically at high frequencies than calculations. Through step 11, the damping coefficient is modified and associated error is reduced to less than $2 \%$.

\section{Results and Discussion}

Model parameters for computing the damping coefficient and stifness are given in Table 1. First $E^{\prime}$ and $E^{\prime \prime}$ are modeled and their simulation together with the experimental results are ploted in Fig. 3 and Fig. 4, respectively. Moreover, the natural frequency of 
the SMPF is estimated in Fig. 5. Then, the values of the damping coefficinet, Fig. 6, and stifness, Fig. 7, are calculated and optimized using displacement results obtained from the experiment. The equation of motion is solved and the displacement at different frequencies before and after parameter optimzation is depicted in Fig. 8.

Table 1 Parameters used to calculate stifness and damping coefficient

\begin{tabular}{|l|l|l|l|l|c|}
\hline Model parameter & $E_{0 T}^{\prime}(\mathrm{MPa})$ & $E_{E G T}^{\prime}(\mathrm{MPa})$ & $E_{h T}^{\prime}(\mathrm{MPa})$ & $E_{1 f}^{\prime}(\mathrm{MPa})$ & $\beta$ \\
\hline$k=\frac{E^{\prime} A}{L}$ & 3000 & 2100 & 20 & 1900 & 0.18 \\
\hline Model parameter & $E_{0 T}^{\prime \prime}(\mathrm{MPa})$ & $E_{E G T}^{\prime \prime}(\mathrm{MPa})$ & $E_{h T}^{\prime \prime}(\mathrm{MPa})$ & $E_{0 f}^{\prime \prime}(\mathrm{MPa})$ & $\gamma$ \\
\hline$c=2 m\left(\sqrt{\left(\frac{k}{m}\right)}\right) \times \frac{E^{\prime}}{E^{\prime \prime}}$ & 9000 & 3400 & 40 & 2720 & 0.08 \\
\hline
\end{tabular}

Table 1 summarizes the parameters related to the Equations 5, 6, 7, and 8; they are introduced to calculate stiffness, $\mathrm{k}$, and damping, $\mathrm{c}$, for the SMPF. The glass transition of the SMPF is $25^{\circ} \mathrm{C}$, which has been reported in the literature [59].

The modeling results for storage modulus and loss modulus are shown in Fig. 3 and Fig. 4, respectively. The results provide an insight into defining the optimal vibration-damping range, which is $7 \mathrm{~Hz}-70 \mathrm{~Hz}$. At this range, maximum energy absorption and damping capability are achieved; this frequency range activates all chains to dissipate energy through entropic elastic mechanism for obtaining random configuration. At frequencies beyond $70 \mathrm{~Hz}$, chains absorb energy of vibration to relax their structure. When the frequency of the vibration reaches to $80 \mathrm{~Hz}$, increased mobility of chains may lead to the fatigue initiation at the hard domain. The illustrated drop in the amount of loss modulus at $100 \mathrm{~Hz}$, in particular for SMPF tested at temperature of $50^{\circ} \mathrm{C}$, may be an outcome of this phenomena. 


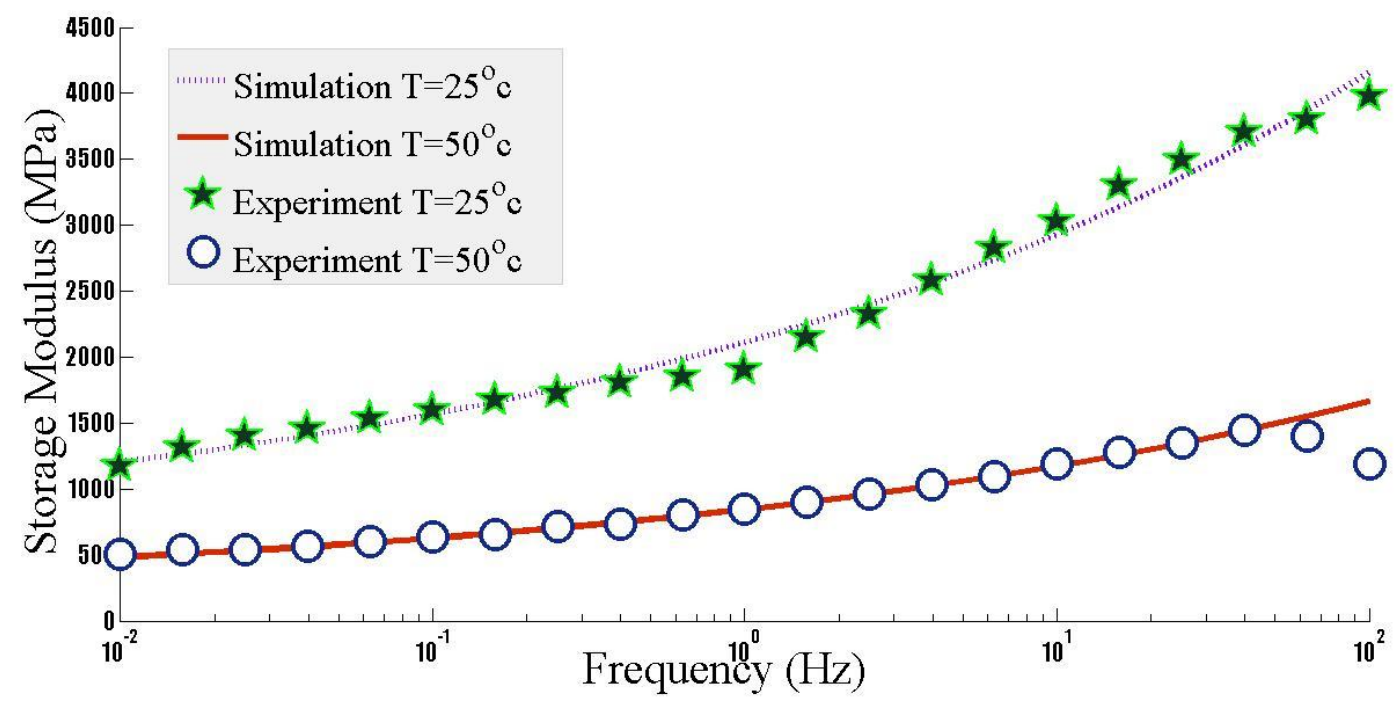

Figure 3 Variation of the storage modulus vs. temperature and frequency.

Damping capability of SMPFs can be tailored by changing the working temperature as well as cold strain and hot programming, which will be discussed in our next paper. Test at a higher temperature, $50^{\circ} \mathrm{C}$, are also shown in Fig. 3 and Fig. 4 . At this temperature, chains are more softened and they tend to relax faster; the overall $40 \%$ drop for storage capability as depicted in Fig. 3 is its ramification. This implies that less than half of chains are saturated at higher temperatures. The greater number of saturated chains resulted from higher capability of the specimen to absorb energy of vibration. However, as illustrated in Fig. 4, the SMPF at higher temperature shows 20\% drop in loss modulus for frequencies lower than $1 \mathrm{~Hz}$. This indicates that the samples at higher frequencies are more compliant and therefore, it is predictable that the loss factor as a measure of damping capability will improve. Moreover, drop in loss modulus shows pronounced effects at higher frequencies (over $1 \mathrm{~Hz}$ ). This excessive drop is related to extended step time in which chains are able to absorb more heat and extensively soften; as a result, the highest amounts of saturated chains are obtained. The saturated chains are the category of polymer chains which are no longer able to regain their original shape and they are in the state of continuous creep.

For both typical sample results at higher frequencies, relaxation time is shorter and chains are at stiffer state compared to that at the lower frequencies. However, the increased internal friction among them results in the initiation of rubbery flow state and 
may lead to the inception of fatigue in the hard domain as well. In other words, at higher frequencies chains should move faster in order to respond to the imposed frequency. Therefore, chains respond as if they are instigated at higher temperatures [38]; hence, activation of structural relaxation phenomenon results in $40 \%$ increase in overall loss factor for the typical sample at higher temperature which is depicted in the Fig. 5.

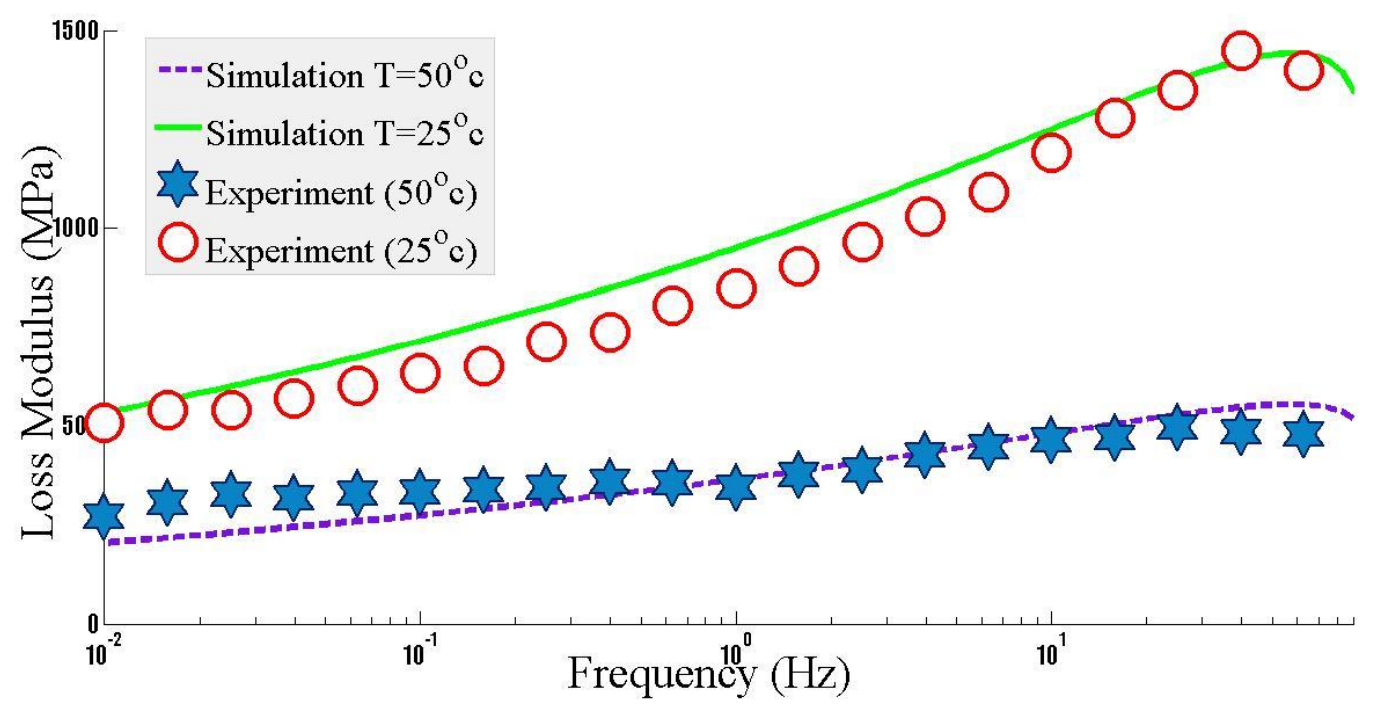

Figure 4 Effect of temperature and frequency on loss modulus of SMPF.

Loss factor vs. log frequency is shown in the Fig. 5, which offers an interesting property related to the observed peak for this plot. The peak damping of $\tan \delta$ occurs at natural frequency of the material which is a function of temperature [38]. Energy of vibration is usually dissipated in the form of heat. Loss factor is defined as the ratio of the imaginary part to the real part of modulus in the frequency domain analysis. However, this concept is equivalent to the energy dissipated per cycle to the energy stored in the material. When the loss factor, i.e., loss tangent plots logarithmically over the span of the frequency sweep test, it illustrates the rate of decay for the amplitude of vibration. At natural frequency, this rate is higher than other frequencies.

Every continuum matter has multiple modes of frequencies that can be a function of temperature and geometry of the specimen. SMPF is a continuum polymer solid matter that can vibrate at a series of distinct frequencies called natural modes, i.e., transition modes. At $50^{\circ} \mathrm{C}$, a typical SMPF sample tends to strongly damp energy of vibration and therefore at least two major peaks are identified, when the natural 
frequency of some segments of the polymer chains is met $(0.063 \mathrm{~Hz}$ and $6.3 \mathrm{~Hz})$. At these frequencies, the chains damp the perturbation energy; inception of structural relaxation of chains at higher temperatures rearranges the microstructure of the SMPF, which results in higher damping as shown in the Fig. 5.

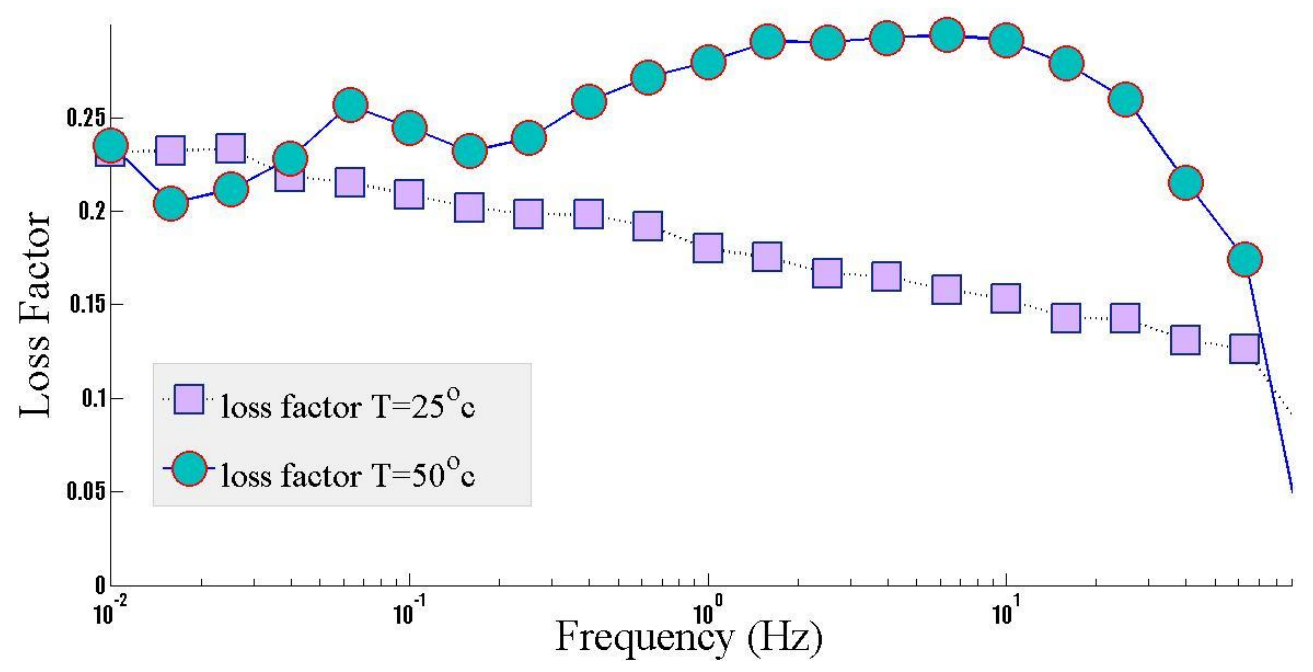

Figure 5 Change of $\tan \delta$ with temperature and frequency

In the free vibration analysis, the ratio of decay of amplitude of vibration is defined as logarithmic decrement, $\Delta$, which is a measure of energy dissipation in the time domain analysis:

$\Delta=\frac{1}{n} \log \left(\frac{x_{i}}{x_{i+1}}\right)$

where $\mathrm{n}$ is the number of cycles and $\mathrm{T}$ is the period of vibration. The relation between loss factor, i.e., $\tan \delta$ and logarithmic decrement is given by [61]:

$\tan \delta=\frac{\Delta}{\pi\left(1+\frac{\Delta^{2}}{4 \pi^{2}}\right)}$

At lower vibration decay, $\Delta^{2}$ is negligible; therefore, one may conclude $\Delta=\pi \tan \delta$. As a result, the higher the tan delta, the greater the damping coefficient, and the more efficient the material will be in effectively accomplishing energy absorption and dissipation. Consequently, the vibration will have lower amplitudes.

The model parameters, damping coefficient and stiffness, have been obtained from the proposed model (shown in Fig. 3 and Fig. 4), and the equation of motion has been solved and their values have been calibrated using the displacement from the 
experiment. The corresponding error is obtained and incorporated into the model to modify the values of the stiffness and damping. Damping and stiffness quantities at different frequencies are calculated and reported. The results of the numerical optimization process as well as original simulation results are shown in Fig. 6 and Fig. 7, respectively. From them, it is seen that the modeling framework has minor deviations for the whole range of simulated frequencies. However, when polymer chains start to saturate, i.e., no longer store or damp the vibration, the optimization process effectively tunes the model's response and eliminates the associated error.

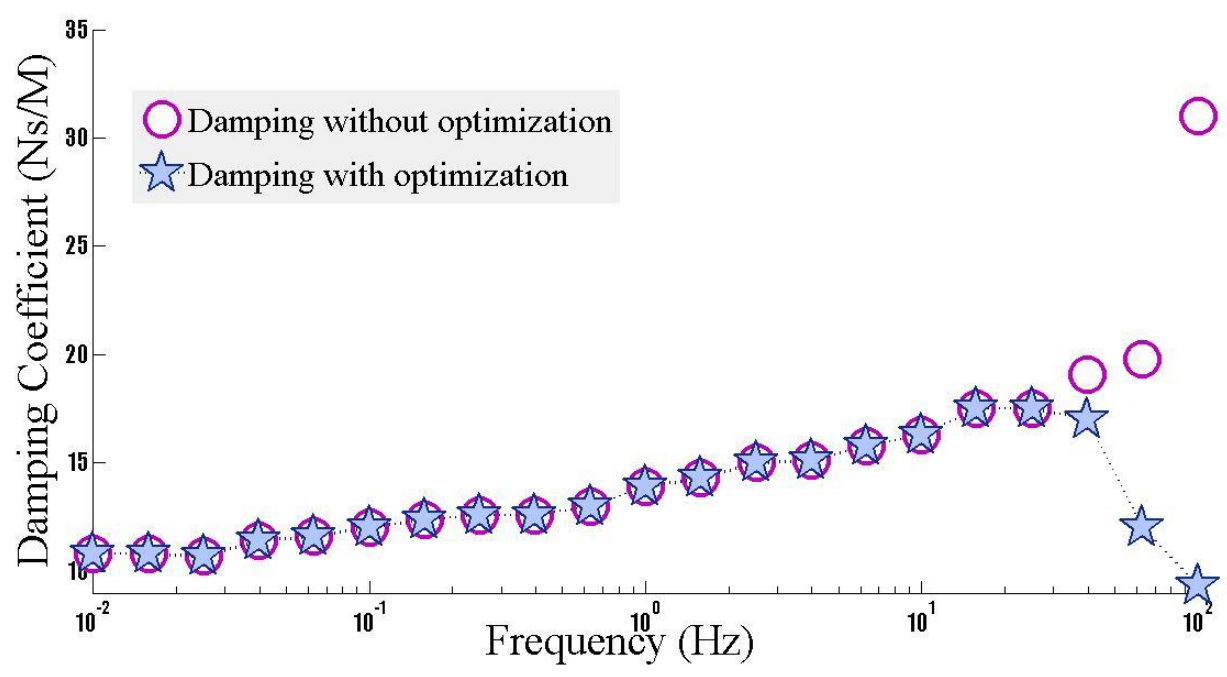

Figure 6 Damping coefficient at different frequencies at $25^{\circ} \mathrm{C}$

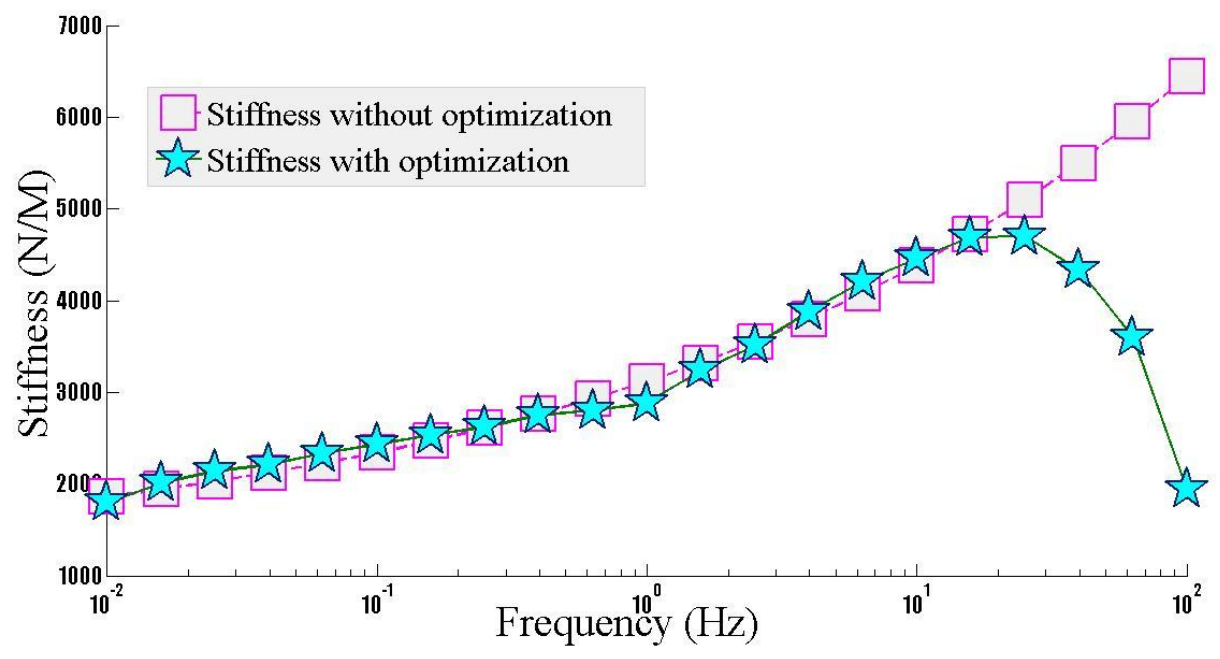

Figure 7 Stiffness change with frequency at $25^{\circ} \mathrm{C}$ 
Stifness and damping quantities obtained from Figs. 6 and 7 are used to solve the equation of motion. The coresponding results are compared with experimental results. The dispalcment simulated with the parameter obtimization is then used to modify the model. Together with the experimetal results, the simulation result is shown in Fig. 8.

Thourough investigations show that the proposed model is capable of reproducing the test results after paramter optimizations with the error less than 5\%.

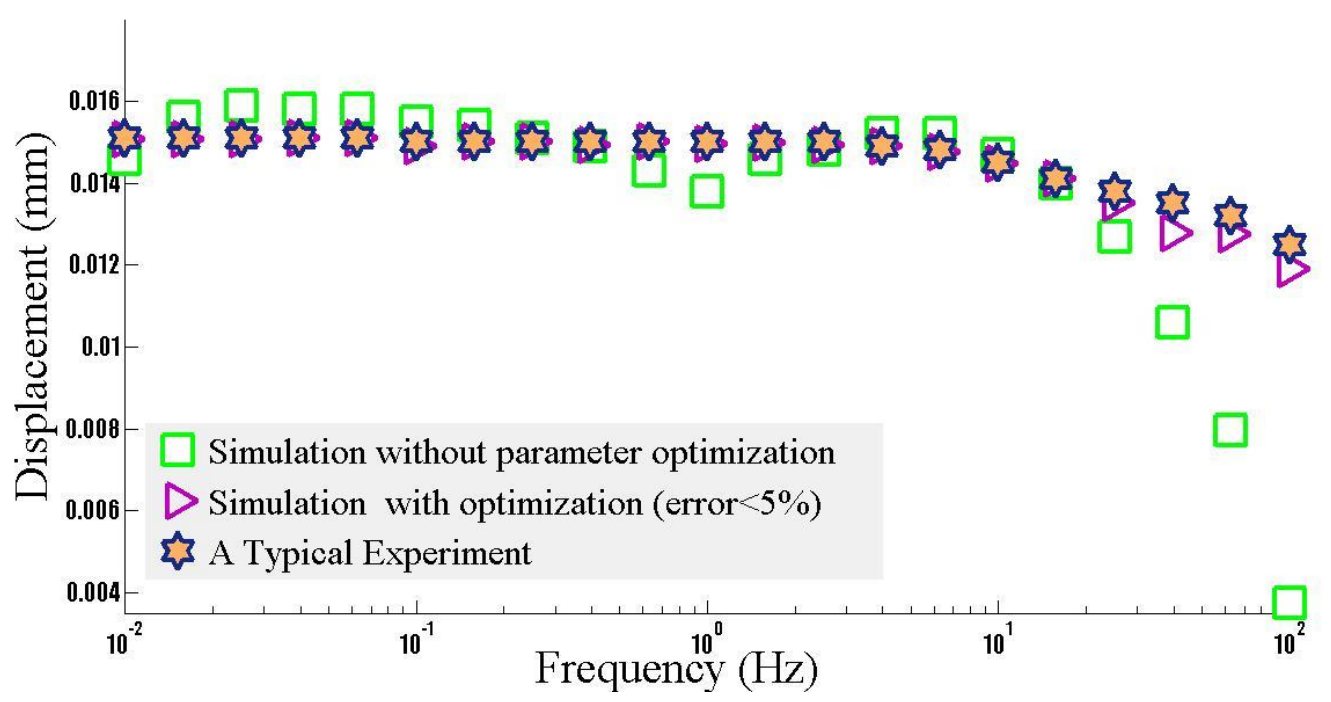

Figure 8 Displacement with and without error mitigation

\section{Conclusion}

In this paper, the dynamic response of a typical polyurethane shape memory polymer fiber has been investigated at two temperatures $25^{\circ} \mathrm{C}$ and $50^{\circ} \mathrm{C}$. The presented experimental data for the SMPF and the numerical model provide an insight into vibration damping application of the SMPF, which has potential to be used in smart composite structures. It is found that

1- The damping capability of SMPFs changes with temperature and frequency.

2- The optimum design region of the SMPFs, with cross section area comparable to the diameter of human hair, is between $7 \mathrm{~Hz}-70 \mathrm{~Hz}$. Within this range, SMPF shows both high storage modulus and damping, which can be opted as a design range.

3- The dynamic storage modulus, obtained from DMA results, can be translated to the stiffness of the SMPF system. In addition the loss factor obtained from 
DMA test results can be correlated to the damping coefficient. Both stiffness and damping properties are critical material properties in the SDOF equation of motion to study vibrational damping response of SMPFs.

It is noted that the proposed methodology is among one of the first attempts to correlate stiffness and damping property of the SMPF to the DMA data. In fact, the damping coefficient and the stiffness of every continuum system under dynamic loading can be reproduced using DMA test results in combination with Eqs. (5) and (6). The proposed methodology provides the design engineers with an analysis tool to study dynamic response of a temperature and frequency dependent system.

\section{Acknowledgement}

This study was financially supported by National Science Foundation under grant number CMMI 1333997, and Army Research Office under grant number W911NF-13-10145. We would like to gratitude valuable comments that Dr. Amir Shojaei has given to improve the quality of the paper and also we would like to thank Ms. Qianxi Yang and Mr. Anqi Wang for their assistance in conducting the experiment.

\section{References:}

1. Liu, C., H. Qin, and P.T. Mather, Review of progress in shape-memory polymers. Journal of Materials Chemistry, 2007. 17(16): p. 1543-1558.

2. $\quad$ Leng J., L.X., Liu Y., Du S., Shape-memory polymers and their composites: stimulus methods and applications. Progress in Materials Science, 2011. 56: p. 1077-1135.

3. Xie, T., Recent advances in polymer shape memory. Polymer Engineering \& Science, 2011. 52(22): p. 4985-5000.

4. Jinlian, H., et al., A review of stimuli-responsive polymers for smart textile applications. Smart Materials and Structures, 2012. 21(5): p. 053001.

5. Meng, H. and G. Li, A review of stimuli-responsive shape memory polymer composites. Polymer, 2013. 54(9): p. 2199-2221.

6. Hager, M.D., et al., Shape memory polymers: Past, present and future developments. Progress in Polymer Science, (0).

7. Jinlian Hu, Y.Z., Huahua Huang, Jing Lu, , Recent advances in shape-memory polymers: Structure, mechanism, functionality, modeling and applications. Progress in Polymer Science, 2012. 37(12): p. 1720-1763.

8. Zhang, P. and G. Li, Structural relaxation behavior of strain hardened shape memory polymer fibers for self-healing applications. Journal of Polymer Science Part B: Polymer Physics, 2013. 51(12): p. 966-977.

9. Zhang, P. and G. Li, Healing-on-demand composites based on polymer artificial muscle. Polymer, 2015. 64(0): p. 29-38. 
10. Yang, Q. and G. Li, Investigation into stress recovery behavior of shape memory polyurethane fiber. Journal of Polymer Science Part B: Polymer Physics, 2014. 52(21): p. 1429-1440.

11. Yang Qianxi, L.G., Spider-silk-like shape memory polymer fiber for vibration damping. Smart Materials and Structures, 2014. 23(10): p. 105032.

12. Li, G., O. Ajisafe, and H. Meng, Effect of strain hardening of shape memory polymer fibers on healing efficiency of thermosetting polymer composites. Polymer, 2013. 54(2): p. 920-928.

13. Li, G., H. Meng, and J. Hu, Healable thermoset polymer composite embedded with stimuli-responsive fibres. Journal of The Royal Society Interface, 2012. 9(77): p. 3279-3287.

14. $\mathrm{Li}, \mathrm{G}$. and P. Zhang, A self-healing particulate composite reinforced with strain hardened short shape memory polymer fibers. Polymer, 2013. 54(18): p. 50755086.

15. Li, G. and A. Shojaei, A viscoplastic theory of shape memory polymer fibres with application to self-healing materials. Proceedings of the Royal Society of London A: Mathematical, Physical and Engineering Sciences, 2012. 468(2144): p. 2319-2346.

16. Shojaei, A. and G. Li, Viscoplasticity analysis of semicrystalline polymers: A multiscale approach within micromechanics framework. International Journal of Plasticity, 2013. 42: p. 31-49.

17. Shojaei, A. and G. Li, Thermomechanical constitutive modelling of shape memory polymer including continuum functional and mechanical damage effects. Proceedings of the Royal Society A: Mathematical, Physical and Engineering Science, 2014. 470(2170).

18. Shojaei, A., G. Li, and G.Z. Voyiadjis, Cyclic Viscoplastic-Viscodamage Analysis of Shape Memory Polymers Fibers With Application to Self-Healing Smart Materials. Journal of Applied Mechanics, 2012. 80(1): p. 011014-011014.

19. Sharafi, S. and G. Li, A multiscale approach for modeling actuation response of polymeric artificial muscles. Soft Matter, 2015. 11(19): p. 3833-3843.

20. Nguyen, T.D., Modeling Shape-Memory Behavior of Polymers. Polymer Reviews, 2013. 53(1): p. 130-152.

21. Nguyen, T.D., et al., A thermoviscoelastic model for amorphous shape memory polymers: Incorporating structural and stress relaxation. Journal of the Mechanics and Physics of Solids, 2008. 56(9): p. 2792-2814.

22. Baghani, M., et al., $A$ thermodynamically-consistent $3 \& \# x a 0 ; D$ constitutive model for shape memory polymers. International Journal of Plasticity, 2012. 35(0): p. 13-30.

23. Chen, Y.-C. and D.C. Lagoudas, A constitutive theory for shape memory polymers. Part II: A linearized model for small deformations. Journal of the Mechanics and Physics of Solids, 2008. 56(5): p. 1766-1778.

24. Qi, H.J., et al., Finite deformation thermo-mechanical behavior of thermally induced shape memory polymers. Journal of the Mechanics and Physics of Solids, 2008. 56(5): p. 1730-1751. 
25. Tobushi, H., et al., Thermomechanical Constitutive Modeling in Shape Memory Polymer of Polyurethane Series. Journal of Intelligent Material Systems and Structures, 1997. 8(8): p. 711-718.

26. Tobushi, $\mathrm{H}$., et al., Thermomechanical constitutive model of shape memory polymer. Mechanics of Materials, 2001. 33(10): p. 545-554.

27. Diani, J., Gall, K., Molecular dynamics simulations of the shape-memory behaviour of polyisoprene. Smart Materials and Structures, 2007: p. 16.

28. Diani, J., et al., Predicting thermal shape memory of crosslinked polymer networks from linear viscoelasticity. International Journal of Solids and Structures, 2012. 49(5): p. 793-799.

29. Diani, J., Y. Liu, and K. Gall, Finite strain 3D thermoviscoelastic constitutive model for shape memory polymers. Polymer Engineering \& Science, 2006. 46(4): p. 486-492.

30. Ge, Q., et al., A finite deformation thermomechanical constitutive model for triple shape polymeric composites based on dual thermal transitions. International Journal of Solids and Structures, 2014. 51(15-16): p. 27772790.

31. Guo, X., et al., Constitutive model for shape memory polymer based on the viscoelasticity and phase transition theories. Journal of Intelligent Material Systems and Structures, 2015.

32. $\mathrm{Li}, \mathrm{G}$. and W. Xu, Thermomechanical behavior of thermoset shape memory polymer programmed by cold-compression: Testing and constitutive modeling. Journal of the Mechanics and Physics of Solids, 2011. 59(6): p. 1231-1250.

33. Liu, Y., et al., Thermomechanics of shape memory polymers: Uniaxial experiments and constitutive modeling. International Journal of Plasticity, 2006. 22(2): p. 279-313.

34. $\mathrm{Xu}, \mathrm{W}$. and $\mathrm{G}$. Li, Constitutive modeling of shape memory polymer based selfhealing syntactic foam. International Journal of Solids and Structures, 2010. 47(9): p. 1306-1316.

35. $\mathrm{Xu}, \mathrm{W}$. and G. Li, Thermoviscoplastic Modeling and Testing of Shape Memory Polymer Based Self-Healing Syntactic Foam Programmed at Glassy Temperature. Journal of Applied Mechanics, 2011. 78(6): p. 061017-061017.

36. Yu, K., Q. Ge, and H.J. Qi, Reduced time as a unified parameter determining fixity and free recovery of shape memory polymers. Nat Commun, 2014. 5.

37. Yu, K., et al., Mechanisms of multi-shape memory effects and associated energy release in shape memory polymers. Soft Matter, 2012. 8(20): p. 5687-5695.

38. Sperling, L.H., Sound and vibration damping with polymers- Basic viscoelastic definitions and concepts., in Sound and Vibration Damping with Polymers, L.H.S. Robert D. Corsaro1, Editor. 1990, ACS Publications. p. 5-22.

39. Bert, C.W., Material damping: An introductory review of mathematic measures and experimental technique. Journal of Sound and Vibration, 1973. 29(2): p. 129-153.

40. Crandall, S.H., The role of damping in vibration theory. Journal of Sound and Vibration, 1970. 11(1): p. 3-IN1.

41. Treviso, A., et al., Damping in composite materials: Properties and models. Composites Part B: Engineering, 2015. 78: p. 144-152. 
42. Tsai, J.-L. and Y.-K. Chi, Effect of fiber array on damping behaviors of fiber composites. Composites Part B: Engineering, 2008. 39(7-8): p. 1196-1204.

43. Lu, N. and S. Oza, A comparative study of the mechanical properties of hemp fiber with virgin and recycled high density polyethylene matrix. Composites Part B: Engineering, 2013. 45(1): p. 1651-1656.

44. Li, X., S.R. Hallett, and M.R. Wisnom, A finite element based statistical model for progressive tensile fibre failure in composite laminates. Composites Part B: Engineering, 2013. 45(1): p. 433-439.

45. Haddad, Y.M. and J. Feng, On the trade-off between damping and stiffness in the design of discontinuous fibre-reinforced composites. Composites Part B: Engineering, 2003. 34(1): p. 11-20.

46. Etaati, A., et al., The study of fibre/matrix bond strength in short hemp polypropylene composites from dynamic mechanical analysis. Composites Part B: Engineering, 2014. 62: p. 19-28.

47. Chen, Q. and C. Levy, Vibration analysis and control of flexible beam by using smart damping structures. Composites Part B: Engineering, 1999. 30(4): $\mathrm{p}$. 395-406.

48. Chaturvedi, S.K. and G.Y. Tzeng, Micromechanical modeling of material damping in discontinuous fiber three-phase polymer composites. Composites Engineering, 1991. 1(1): p. 49-60.

49. Nowick, A.S., and B. S. Berry, Anelastic Relaxation in Crystalline Solids. . 1972, New York: Academic.

50. Caughey, T.K. and A. Vijayaraghavan, Free and forced oscillations of a dynamic system with "linear hysteretic damping" (non-linear theory). International Journal of Non-Linear Mechanics, 1970. 5(3): p. 533-555.

51. Bagley, R.L. and J. Torvik, Fractional calculus - A different approach to the analysis of viscoelastically damped structures. AIAA Journal, 1983. 21(5): p. 741-748.

52. Johnson, B.E. and A. Hoger, The Use of Strain Energy to Quantify the Effect of Residual Stress on Mechanical Behavior. Mathematics and Mechanics of Solids, 1998. 3(4): p. 447-470.

53. McTavish D. J., H.P.C., Modeling of Linear Viscoelastic Space Structures. Journal of Vibration and Acoustics, 1993. 115(1): p. 103-110.

54. McTavish., W.C.G.C.A.S.D.J., Implementation of the Golla-Hughes-McTavish (GHM) method for viscoelastic materials using MATLAB and NASTRAN. . Proc. SPIE 2445, Smart Structures and Materials ,Passive Damping., 1995. 312

55. Lesieutre, G.A., Finite elements for dynamic modeling of uniaxial rods with frequency-dependent material properties. International Journal of Solids and Structures, 1992. 29(12): p. 1567-1579.

56. Lesieutre, G.A., E. Bianchini, and A. Maiani, Finite element modeling of onedimensional viscoelastic structures using anelastic displacement fields. Journal of Guidance, Control, and Dynamics, 1996. 19(3): p. 520-527.

57. Rao, S.S., Mechanical vibrations. 1986: Addison-Wesley Longman, Incorporated.

58. Dao, K.C. and D.J. Dicken, Fatigue failure mechanisms in polymers. Polymer Engineering \& Science, 1987. 27(4): p. 271-276. 
59. Li, G., Self-Healing Composites: Shape Memory Polymerbased Structures. 2014: John Wiley \& Sons Ltd.

60. Kreyszig, E., Advanced Engineering Mathematics. 2010: John Wiley \& Sons.

61. 4664-1, I., Rubber, vulcanized or thermoplastic -- Determination of dynamic properties -- Part 1: General guidance. 2005: Switzerland 